\title{
A sufficient condition for the linear stability of magnetohydrodynamic equilibria with field aligned incompressible flows
}

\author{
G. N. Throumoulopoulos \\ University of Ioannina, Association Euratom - Hellenic Republic, \\ Division of Theoretical Physics, GR 45110 Ioannina, Greece \\ H. Tasso \\ Max-Planck-Institut für Plasmaphysik, Euratom Association, \\ D-85748 Garching, Germany
}

\begin{abstract}
A sufficient condition for the linear stability of three dimensional equilibria with incompressible flows parallel to the magnetic field is derived. The condition involves physically interpretable terms related to the magnetic shear and the flow shear.
\end{abstract}




\section{Introduction}

For static ideal magnetohydrodynamic (MHD) equilibria there is a powerful tool known as "the energy principle" providing necessary and sufficient conditions for linear stability [1]. In the presence of flow, however, the stability problem is much tougher because the force operator becomes non Her-

mitian; thus, only sufficient conditions were obtained [2]-[10]. Motivation of the present study is a couple of papers by Ilin and Vladimirov [6, 8] in which a sufficient condition was derived for the linear stability of plasmas with constant density and incompressible flows parallel to the magnetic field. This condition states that an equilibrium is stable to three dimensional perturbations provided that: i) the flow is sub-Alfvenic and ii) inequalities (51) of Ref. [8] are satisfied. Here we show, however, that those inequalities are not correct for the following reasons:

1. The authors of Refs. [6] and [8] have not noticed that because of the field aligned flow the equilibrium current density lies on magnetic surfaces. This property simplifies the stability analysis and results in a single inequality for the sufficient condition in place of the couple of inequalities (51) of Ref. [8].

2. A term associated with the flow shear was ignored in Refs [6] and [8].

The correct sufficient condition obtained here contains physically interpretable terms related to the magnetic shear and the flow shear.

The equilibrium characteristics are examined in Sec. II including a prove of the coincidence of the current density surfaces with the magnetic surfaces. Sec. III reviews the energy principle established in Refs. [6] and [8] which subsequently is employed in Sec. IV to derive the sufficient condition. A major part of the derivation is presented in the Appendix.

\section{Equilibrium}

We consider the steady states of a plasma of constant density and incompressible flow parallel to the magnetic filed in the framework of ideal MHD (see for example Eqs. (1)-(6) of Ref. [11] written in convenient units and the density set to unity). Also, it is assumed the existence of well defined equilibrium magnetic surfaces in three dimensional geometry which are labeled by a smooth function $\psi$. Using

$$
\mathbf{V}=\lambda \mathbf{B}
$$


where $\lambda$ is an arbitrary function, the incompressibility condition $(\boldsymbol{\nabla} \cdot \mathbf{V}=0)$ implies that $\lambda$ is a surface quantity:

$$
\lambda=\lambda(\psi) .
$$

Then, employing the identity $(\mathbf{V} \cdot \boldsymbol{\nabla}) \mathbf{V}=\boldsymbol{\nabla} V^{2} / 2-\mathbf{V} \times \boldsymbol{\nabla} \times \mathbf{V}$, the momentum equation

$$
(\mathbf{V} \cdot \nabla) \mathbf{V}=\mathbf{J} \times \mathbf{B}-\nabla P
$$

leads to

$$
\left(1-\lambda^{2}\right) \mathbf{J} \times \mathbf{B}=\boldsymbol{\nabla}\left(P+\frac{\lambda^{2} B^{2}}{2}\right)-B^{2} \boldsymbol{\nabla}\left(\frac{\lambda^{2}}{2}\right),
$$

where $B$ is the magnetic field modulus. The component of (3) along the magnetic field implies that the quantity $P+\lambda^{2} B^{2} / 2$ is uniform on magnetic surfaces:

$$
P+\frac{\lambda^{2} B^{2}}{2} \equiv P_{s}(\psi)
$$

Thus, owing to the flow the isobaric surfaces depart from the magnetic surfaces unlike the case of static equilibrium associated with the surface function $P_{s}(\psi)$. Consequently, Eq. (3) is put in the form

$$
\left(1-\lambda^{2}\right) \mathbf{J} \times \mathbf{B}=P_{s}^{\prime} \nabla \psi-\left(\lambda^{2}\right)^{\prime} \frac{B^{2}}{2} \nabla \psi
$$

or

$$
\mathbf{N} \equiv \mathbf{J} \times \mathbf{B}=g\left(\psi, B^{2}\right) \boldsymbol{\nabla} \psi
$$

where

$$
g\left(\psi, B^{2}\right) \equiv \frac{P_{s}^{\prime}}{1-\lambda^{2}}-\frac{\left(\lambda^{2}\right)^{\prime}}{1-\lambda^{2}} \frac{B^{2}}{2} .
$$

Eq. (5) implies that the current density lies on magnetic surfaces a property not noticed in Refs. [6] and [8]. Note that this holds because of the incompressible field aligned flows; for flows of arbitrary direction the current surfaces do not coincide with the magnetic surfaces. The fact that $\mathbf{B}, \mathbf{J}$ and V share the same surfaces simplify the stability analysis to follow. To this end we also will need the quantity

$$
\mathbf{M} \equiv \nabla \times \mathbf{N}=\nabla g \times \nabla \psi
$$

from which it follows that

$$
\mathbf{M} \cdot \mathbf{N}=0 .
$$




\section{Review of the energy principle}

In Refs. [6, 8] an energy principle was established for incompressible perturbations $[\boldsymbol{\nabla} \cdot \boldsymbol{\xi}(\mathbf{x}, t)=0]$ around a steady state and non-slip boundary conditions:

$$
\mathbf{v} \cdot \mathbf{n}=\mathbf{b} \cdot \mathbf{n}=\boldsymbol{\xi} \cdot \mathbf{n}=0 .
$$

Here $\mathbf{v}(\mathbf{x}, t)$ and $\mathbf{b}(\mathbf{x}, t)$ are the perturbations of the velocity and the magnetic field and conditions (9) are imposed on a fixed boundary $\partial \mathcal{D}$ surrounding the plasma domain $\mathcal{D}$. The principle is based on the fact that the perturbation energy

$$
E \equiv \int_{\mathcal{D}}\left(\frac{1}{2} \dot{\boldsymbol{\xi}}^{2}-\frac{1}{2} \boldsymbol{\xi} \cdot \hat{K} \boldsymbol{\xi}\right) d V
$$

is conserved by the linearized ideal MHD equations $(d E / d t=0)$. Here $\hat{K}$ is a symmetric operator defined by the formula

$$
\hat{K} \boldsymbol{\xi}=\mathbf{V} \times \nabla \times \mathbf{v}+\mathbf{v} \times \Omega-\mathbf{B} \times \nabla \times \mathbf{b}-\mathbf{b} \times \mathbf{J},
$$

where $\mathbf{v}=\boldsymbol{\nabla} \times(\boldsymbol{\xi} \times \mathbf{V})$,

$$
\mathbf{b}=\nabla \times(\xi \times \mathbf{B})
$$

and $\boldsymbol{\Omega}=\boldsymbol{\nabla} \times \mathbf{V}$. Evidently, $E$ as a quadratic functional of $\dot{\boldsymbol{\xi}}$ and $\boldsymbol{\xi}$ is positive definite if the potential energy

$$
W=-\frac{1}{2} \int_{\mathcal{D}} \boldsymbol{\xi} \cdot \hat{K} \boldsymbol{\xi} d V
$$

is positive definite. It is known, however, that for flows of arbitrary direction the functional $W$ is never strictly positive definite [2]-[10]. For this reason further consideration is restricted to the steady states with field aligned flows described in Sec. II. In this case (12) can be written in the form

$$
W=\frac{1}{2} \int_{\mathcal{D}}\left\{\left(1-\lambda^{2}\right)\left[\mathbf{b}^{2}+\mathbf{b} \cdot(\mathbf{J} \times \boldsymbol{\xi})\right]-2 \lambda(\boldsymbol{\xi} \cdot \nabla \lambda)[\boldsymbol{\xi} \cdot(\mathbf{B} \cdot \boldsymbol{\nabla}) \mathbf{B}]\right\} d V .
$$

Derivation of (13) is given in Ref. 6]. Whenever the potential energy (13) is positive definite the equilibrium is linearly stable.

\section{Sufficient condition for linear stability}


As in Refs. [6] and [8] assuming that $\mathbf{J} \times \mathbf{B} \neq 0$ we express the perturbation vector $\boldsymbol{\xi}$ in the form

$$
\boldsymbol{\xi}=\alpha(\mathbf{x}, t) \mathbf{N}+\beta(\mathbf{x}, t) \mathbf{J}+\gamma(\mathbf{x}, t) \mathbf{B} .
$$

It can then be shown (see Appendix) that $W$ assumes the form

$$
\begin{gathered}
W=W_{1}+W_{2}, \\
W_{1}=\frac{1}{2} \int_{\mathcal{D}}\left(1-\lambda^{2}\right)(\mathbf{b}+\alpha \mathbf{J} \times \mathbf{N})^{2} d V, \\
W_{2}=\int_{\mathcal{D}} A \alpha^{2},
\end{gathered}
$$

where

$$
A=-\left(1-\lambda^{2}\right)(\mathbf{J} \times \mathbf{N}) \cdot(\mathbf{B} \cdot \boldsymbol{\nabla}) \mathbf{N}-\lambda(\mathbf{N} \cdot \boldsymbol{\nabla} \lambda)\left(\mathbf{N} \cdot \frac{\boldsymbol{\nabla} B^{2}}{2}+N^{2}\right) .
$$

Evidently, $W$ is positive semidefinite if $|\lambda| \leq 1$ and

$$
A \geq 0 \text { in } \mathcal{D} \text {. }
$$

Inequality (19) is substantially different from the respective inequalities (51) of Ref. 8. In particular, the last term of (18) containing $\nabla \lambda$ was missed in [6] and [8]. Using the equilibrium relations of Sec. III, (18) reduces to

$$
\begin{aligned}
A= & -g^{2}\left\{(1-\lambda)^{2}(\mathbf{J} \times \boldsymbol{\nabla} \psi) \cdot(\mathbf{B} \cdot \boldsymbol{\nabla}) \boldsymbol{\nabla} \psi\right. \\
& \left.-\frac{\left(\lambda^{2}\right)^{\prime}}{2}|\boldsymbol{\nabla} \psi|^{2}\left(\boldsymbol{\nabla} \psi \cdot \frac{\boldsymbol{\nabla} B^{2}}{2}+g|\boldsymbol{\nabla} \psi|^{2}\right)\right\} .
\end{aligned}
$$

On account of (15)-(17) and (20) we can conclude that a general steady state of a plasma of constant density and incompressible flows parallel to the magnetic is stable to small three-dimensional perturbations if i) the flow is sub-Alfvénic and ii)

$$
\tilde{A} \equiv \frac{A}{g^{2}} \geq 0 .
$$

Using the relation

$$
(\mathbf{B} \cdot \boldsymbol{\nabla}) \boldsymbol{\nabla} \psi=\mathbf{J} \times \boldsymbol{\nabla} \psi-(\boldsymbol{\nabla} \psi \cdot \boldsymbol{\nabla}) \mathbf{B}
$$


$\tilde{A}$ can be put in the physically interpretable form:

$$
\begin{aligned}
\tilde{A}= & -(1-\lambda)^{2}\left[(\mathbf{J} \times \boldsymbol{\nabla} \psi)^{2}-(\mathbf{J} \times \boldsymbol{\nabla} \psi) \cdot(\boldsymbol{\nabla} \psi \cdot \boldsymbol{\nabla}) \mathbf{B}\right] \\
& -\frac{\left(\lambda^{2}\right)^{\prime}}{2}|\boldsymbol{\nabla} \psi|^{2}\left(\boldsymbol{\nabla} \psi \cdot \frac{\boldsymbol{\nabla} B^{2}}{2}+g|\boldsymbol{\nabla} \psi|^{2}\right) .
\end{aligned}
$$

The first negative destabilizing term in (22) should be related to current driven modes. The other terms can be either stabilizing or destabilizing. This depends on the sign of $\left(\lambda^{2}\right)^{\prime}$ in relation to the velocity shear and on the differential variation of $\mathbf{B}$ and $B^{2}$ perpendicular to the magnetic surfaces in relation to the magnetic shear. Also, the last term has an additional implicit dependence on $\left(\lambda^{2}\right)^{\prime}$ and $P_{s}^{\prime}$ through the quantity $g$ [Eq. (6) ].

It is recalled that the sufficient condition established here can be applied to any steady state without geometrical restriction. Application to steady states of fusion concern in connection with possible stabilizing effects of the flow is under way.

\section{Appendix: Derivation of (15)-(18)}

The procedure to follow is based on that of Appendix of Ref. 8]. Since there are substantial differences, however, the derivation will be presented in a self contained way.

Preliminarily, in view of the representation (14) for $\boldsymbol{\xi}$ and the incompressibility condition $\nabla \cdot \boldsymbol{\xi}=0$ we obtain the following relations:

$$
\begin{gathered}
\boldsymbol{\nabla} \cdot(\alpha \mathbf{N})+\mathbf{J} \cdot \boldsymbol{\nabla} \beta+\mathbf{B} \cdot \boldsymbol{\nabla} \gamma=0, \\
\boldsymbol{\xi} \times \mathbf{B}=\alpha \mathbf{N} \times \mathbf{B}+\beta \mathbf{N}, \quad \mathbf{J} \times \boldsymbol{\xi}=\alpha \mathbf{J} \times \mathbf{N}+\gamma \mathbf{N} .
\end{gathered}
$$

Also, (11) becomes

$$
\mathbf{b}=\boldsymbol{\nabla} \times(\alpha \mathbf{N} \times \mathbf{B}+b \mathbf{N})
$$

The first term of (13) is written as

$$
\begin{aligned}
I \equiv & \frac{1}{2} \int_{\mathcal{D}}\left(1-\lambda^{2}\right)\left[\mathbf{b}^{2}+\mathbf{b} \cdot(\mathbf{J} \times \boldsymbol{\xi})\right] d V \\
= & \frac{1}{2} \int_{\mathcal{D}}\left(1-\lambda^{2}\right)\left[\mathbf{b}^{2}+\mathbf{b} \cdot(\alpha \mathbf{J} \times \mathbf{N}+\gamma \mathbf{N})\right] d V \\
= & \frac{1}{2} \int_{\mathcal{D}}\left(1-\lambda^{2}\right)\left[(\mathbf{b}+\alpha \mathbf{J} \times \mathbf{N})^{2}-\alpha^{2}(\mathbf{J} \times \mathbf{N})^{2}\right. \\
& -\mathbf{b} \cdot(\alpha \mathbf{J} \times \mathbf{N})+\mathbf{b} \cdot(\gamma \mathbf{N})] d V
\end{aligned}
$$


Employing the identity $\boldsymbol{\nabla} \cdot(\mathbf{A} \times \mathbf{B})=\mathbf{A} \cdot \nabla \times \mathbf{B}-\mathbf{B} \cdot \boldsymbol{\nabla} \times \mathbf{A}$ we have in connection with the last term of $(\underline{24})$

$$
\begin{aligned}
\mathbf{b} \cdot(\gamma \mathbf{N}) & =\gamma \mathbf{N} \cdot \nabla \times(\alpha \mathbf{N} \times \mathbf{B}+\beta \mathbf{N}) \\
& =(\alpha \mathbf{N} \times \mathbf{B}+\beta \mathbf{N}) \cdot \nabla \times(\gamma \mathbf{N})+\nabla \cdot[(\alpha \mathbf{N} \times \mathbf{B}+\beta \mathbf{N}) \times(\gamma \mathbf{N})]
\end{aligned}
$$

and therefore

$$
\begin{aligned}
\left(1-\lambda^{2}\right) \mathbf{b} \cdot(\gamma \mathbf{N})= & \left(1-\lambda^{2}\right)(\alpha \mathbf{N} \times \mathbf{B}+\beta \mathbf{N}) \cdot \boldsymbol{\nabla} \times(\gamma \mathbf{N}) \\
& +\boldsymbol{\nabla} \cdot\left[\left(1-\lambda^{2}\right) \gamma(\alpha \mathbf{N} \times \mathbf{B}+\beta \mathbf{N}) \times \mathbf{N}\right]
\end{aligned}
$$

the last term following from the fact that $\mathbf{N}$ is proportional to $\boldsymbol{\nabla} \psi$ [Eq. (5)]. Substituting (25) into (24) and integrating by parts furnishes

$$
\begin{aligned}
I= & \frac{1}{2} \int_{\mathcal{D}}\left(1-\lambda^{2}\right)\left[(\mathbf{b}+\alpha \mathbf{J} \times \mathbf{N})^{2}-\alpha^{2}(\mathbf{J} \times \mathbf{N})^{2}\right. \\
& \overbrace{-\alpha(\mathbf{J} \times \mathbf{N}) \cdot \mathbf{b}}^{Y_{2}}+\overbrace{\nabla \times(\gamma \mathbf{N}) \cdot(\alpha \mathbf{N} \times \mathbf{B}+\beta \mathbf{N})}^{Y_{1}}] d V .
\end{aligned}
$$

Furthermore, employing (7) and (8) we find for the above defined quantities $Y_{1}$ and $Y_{2}$ :

$$
\begin{aligned}
Y_{1} & =\boldsymbol{\nabla} \times(\gamma \mathbf{N}) \cdot(\alpha \mathbf{N} \times \mathbf{B}+\beta \mathbf{N})=(\gamma \nabla \times \mathbf{N}+\nabla \gamma \times \mathbf{N}) \cdot(\alpha \mathbf{N} \times \mathbf{B}+\beta \mathbf{N}) \\
& =\alpha \gamma \mathbf{M} \cdot \mathbf{N} \times \mathbf{B}-a N^{2}(\mathbf{B} \cdot \nabla \gamma) \\
Y_{2} & =-\alpha(\mathbf{J} \times \mathbf{N}) \cdot \nabla \times(\alpha \mathbf{N} \times \mathbf{B}+\beta \mathbf{N}) \\
& =-\alpha(\mathbf{J} \times \mathbf{N}) \cdot[\nabla \alpha \times(\mathbf{N} \times \mathbf{B})+\alpha \boldsymbol{\nabla} \times(\mathbf{N} \times \mathbf{B})+\nabla \beta \times \mathbf{N}+\beta \mathbf{M}] \\
& =-\alpha^{2}(\mathbf{J} \times \mathbf{N}) \cdot \nabla \times(\mathbf{N} \times \mathbf{B})-\alpha N^{2}(\mathbf{N} \cdot \nabla \alpha+\mathbf{J} \cdot \nabla \beta)-\alpha \beta \mathbf{M} \cdot(\mathbf{J} \times \mathbf{N})
\end{aligned}
$$

and with the aid of (23)

$$
Y_{1}+Y_{2}=-\alpha^{2}\left[(\mathbf{J} \times \mathbf{N}) \cdot \nabla \times(\mathbf{N} \times \mathbf{B})-N^{2} \boldsymbol{\nabla} \cdot \mathbf{N}\right]-\alpha \beta \mathbf{J} \cdot(\mathbf{N} \times \mathbf{M})-\alpha \gamma \mathbf{B} \cdot(\mathbf{N} \times \mathbf{M}) .
$$

Eq. (25) then becomes

$$
\begin{aligned}
I= & \frac{1}{2} \int_{\mathcal{D}}\left(1-\lambda^{2}\right)\left\{(\mathbf{b}+\alpha \mathbf{J} \times \mathbf{N})^{2}\right. \\
& -\alpha^{2}\left[(\mathbf{J} \times \mathbf{N}) \cdot \boldsymbol{\nabla} \times(\mathbf{N} \times \mathbf{B})-N^{2} \boldsymbol{\nabla} \cdot \mathbf{N}+(\mathbf{J} \times \mathbf{N})^{2}\right] \\
& -\alpha \beta \mathbf{J} \cdot(\mathbf{N} \times \mathbf{M})-\alpha \gamma \mathbf{B} \cdot(\mathbf{N} \times \mathbf{M})\} d V .
\end{aligned}
$$


The staff within the square brackets in (26) can be put in the concise form

$$
(\mathbf{J} \times \mathbf{N}) \cdot \boldsymbol{\nabla} \times(\mathbf{N} \times \mathbf{B})-N^{2} \boldsymbol{\nabla} \cdot \mathbf{N}+(\mathbf{J} \times \mathbf{N})^{2}=2(\mathbf{J} \times \mathbf{N}) \cdot(\mathbf{B} \cdot \boldsymbol{\nabla}) \mathbf{N} .
$$

To show this we employ the relation $\boldsymbol{\nabla}(\mathbf{B} \cdot \mathbf{N})=0$ implying that

$$
(\mathbf{N} \cdot \boldsymbol{\nabla}) \mathbf{B}=-(\mathbf{B} \cdot \boldsymbol{\nabla}) \mathbf{N}+\mathbf{M} \times \mathbf{B}+\mathbf{J} \times \mathbf{N}
$$

then,

$$
\begin{aligned}
(\mathbf{J} \times \mathbf{N}) \cdot \boldsymbol{\nabla} \times(\mathbf{N} \times \mathbf{B}) & =(\mathbf{J} \times \mathbf{N}) \cdot[(\mathbf{B} \cdot \boldsymbol{\nabla}) \mathbf{N}-\mathbf{B}(\boldsymbol{\nabla} \cdot \mathbf{N})-(\mathbf{N} \cdot \boldsymbol{\nabla}) \mathbf{B}] \\
& =2(\mathbf{J} \times \mathbf{N}) \cdot(\mathbf{B} \cdot \boldsymbol{\nabla}) \mathbf{N}-(\mathbf{J} \times \mathbf{N})^{2}+N^{2} \boldsymbol{\nabla} \cdot \mathbf{N} .
\end{aligned}
$$

We now consider the second part of $W$ [see Eq. (13)] :

$$
Q \equiv=-\int_{\mathcal{D}} \lambda(\boldsymbol{\xi} \cdot \boldsymbol{\nabla} \lambda)[\boldsymbol{\xi} \cdot(\mathbf{B} \cdot \boldsymbol{\nabla}) \mathbf{B}] d V .
$$

Using the relations

$$
(\boldsymbol{\xi} \cdot \lambda)=\alpha \mathbf{N} \cdot \nabla \lambda
$$

and

$$
\boldsymbol{\xi} \cdot(\mathbf{B} \cdot \boldsymbol{\nabla}) \mathbf{B}=\boldsymbol{\xi} \cdot\left(\frac{\boldsymbol{\nabla} B^{2}}{2}+\mathbf{J} \times \mathbf{B}\right)=(\alpha \mathbf{N}+\beta \mathbf{J}+\gamma \mathbf{B}) \cdot \frac{\boldsymbol{\nabla} B^{2}}{2}+\alpha N^{2},
$$

(28) is put in the form

$$
\begin{aligned}
Q= & -\int_{\mathcal{D}}\left\{\lambda \alpha^{2}(\mathbf{N} \cdot \boldsymbol{\nabla} \lambda)\left(\mathbf{N} \cdot \frac{\boldsymbol{\nabla} B^{2}}{2}+N^{2}\right)\right. \\
& \left.+\lambda \alpha \beta(\mathbf{N} \cdot \boldsymbol{\nabla} \lambda)\left(\mathbf{J} \cdot \frac{\boldsymbol{\nabla} B^{2}}{2}\right)+\lambda \alpha \gamma(\mathbf{N} \cdot \boldsymbol{\nabla} \lambda)\left(\mathbf{B} \cdot \frac{\boldsymbol{\nabla} B^{2}}{2}\right)\right\} d V
\end{aligned}
$$

The first term in (29) containing $\nabla \lambda$ was ignored in Refs. [6] and [8]. In view of (26), (27) and (29), $W$ is written in the form

$$
\begin{gathered}
W=W_{1}+W_{2}, \\
W_{1}=\frac{1}{2} \int_{\mathcal{D}}\left(1-\lambda^{2}\right)(\mathbf{b}+\alpha \mathbf{J} \times \mathbf{N})^{2} d V,
\end{gathered}
$$




$$
\begin{aligned}
W_{2}= & \frac{1}{2} \int_{\mathcal{D}}\left\{-2 \alpha^{2}\left[\left(1-\lambda^{2}\right)(\mathbf{J} \times \mathbf{N}) \cdot(\mathbf{B} \cdot \boldsymbol{\nabla}) \mathbf{N}\right.\right. \\
& \left.+\lambda(\mathbf{N} \cdot \boldsymbol{\nabla} \lambda)\left(\mathbf{N} \cdot \frac{\boldsymbol{\nabla} B^{2}}{2}+N^{2}\right)\right] \\
& -\alpha \beta\left[\left(1-\lambda^{2}\right) \mathbf{J} \cdot(\mathbf{N} \times \mathbf{M})+2 \lambda(\mathbf{N} \cdot \boldsymbol{\nabla} \lambda)\left(\mathbf{J} \cdot \frac{\boldsymbol{\nabla} B^{2}}{2}\right)\right] \\
& \left.-\alpha \gamma\left[\left(1-\lambda^{2}\right) \mathbf{B} \cdot(\mathbf{N} \times \mathbf{M})+2 \lambda(\mathbf{N} \cdot \boldsymbol{\nabla} \lambda)\left(\mathbf{B} \cdot \frac{\boldsymbol{\nabla} B^{2}}{2}\right)\right]\right\} d V .
\end{aligned}
$$

The coefficients of $\alpha \beta$ and $\alpha \gamma$ vanish identically. Indeed, on account of the equilibrium relations (21), (44), (5) and (6) we have for the coefficient of $\alpha \beta$ :

$$
\begin{aligned}
& \left(1-\lambda^{2}\right) \mathbf{J} \cdot(\mathbf{N} \times \mathbf{M})+2 \lambda(\mathbf{N} \cdot \boldsymbol{\nabla} \lambda)\left(\mathbf{J} \cdot \frac{\boldsymbol{\nabla} B^{2}}{2}\right) \\
& =\left(1-\lambda^{2}\right) g|\boldsymbol{\nabla} \psi|^{2} \mathbf{J} \cdot \boldsymbol{\nabla} g+\left(\lambda^{2}\right)^{\prime} g|\boldsymbol{\nabla} \psi|^{2} \mathbf{J} \cdot \frac{\boldsymbol{\nabla} B^{2}}{2} \\
& =g|\boldsymbol{\nabla} \psi|^{2}\left\{\mathbf{J} \cdot \boldsymbol{\nabla}\left[\left(1-\lambda^{2}\right) g\right]+\mathbf{J} \cdot \boldsymbol{\nabla} \cdot\left[\left(\lambda^{2}\right)^{\prime} \frac{B^{2}}{2}\right]\right\}=0 .
\end{aligned}
$$

The coefficient of $\alpha \gamma$ also vanishes because is symmetric to the coefficient of $\alpha \beta$ in replacing $\mathbf{J}$ with $\mathbf{B}$. Consequently (32) assumes the form (17). 


\section{Acknowledgements}

Part of this work was conducted during a visit of the author G.N.T. to the Max-Planck-Institut für Plasmaphysik, Garching. The hospitality of that Institute is greatly appreciated.

This work was performed within the participation of the University of Ioannina in the Association Euratom-Hellenic Republic, which is supported in part by the European Union and by the General Secretariat of Research and Technology of Greece. The views and opinions expressed herein do not necessarily reflect those of the European Commission. 


\section{References}

[1] I. B. Bernstein, E. A. Frieman, M. D. Kruskul, R. M. Kulsrud, Proc. Roy. Soc. (London), A244, 17 (1958).

[2] E. Frieman and M. Rotenberg, Rev. Mod. Phys. 32, 898 (1960).

[3] S. Friedlander, M. M. Vishik, Chaos 5, 416 (1995).

[4] H. Tasso, Phys. Lett. A 222, 97 (1996).

[5] E. Hameiri, Phys. Plasmas 5, 3270 (1998).

[6] V. A. Vladimirov and K. I. Ilin, Phys. Plasmas 5, 4199 (1998).

[7] A. V. Kats JETP Letters 77, 657 (2003).

[8] K. I. Ilin and V. A. Vladimirov, Phys. Plasmas 11, 3586 (2004).

[9] V. I. Ilgisonis and I. V. Khalzov JETP Letters 82, 570 (2005).

[10] M. Hirota, Z. Yoshida, E. Hameiri Phys. Plasmas 13, 022107 (2006).

[11] H. Tasso and G. N. Throumoulopoulos, Phys. Plasmas 8, 2378 (1998). 